\title{
ON SOME TWO WAY CLASSIFICATIONS OF INTEGERS*
}

\author{
J. Lambek and L. Moser
}

(received February 26, 1959)

In this note we use the method of generating functions to show that there is a unique way of splitting the non-negative integers into two classes in such a way that the sums of pairs of distinct integers will be the same (with same multiplicities) for both classes. We prove a similar theorem for products of positive integers and consider some related problems.

Suppose then that the non-negative integers are split into two classes $A$ and $B$ with

$$
\begin{aligned}
& \mathrm{A}: 0=\mathrm{a}_{1}<\mathrm{a}_{2}<\ldots . \\
& \mathrm{B}: \quad \mathrm{b}_{1}<\mathrm{b}_{2}<\ldots .
\end{aligned}
$$

Consider the corresponding generating functions

$$
\begin{aligned}
& A \leftrightarrow f(x)=\sum_{i} x^{a_{i}}, \quad|x|<1 . \\
& B \leftrightarrow g(x)=\sum_{j} x^{b_{j}}, \quad|x|<1 .
\end{aligned}
$$

Since every non-negative integer belongs to exactly one class we have

$$
f(x)+g(x)=1 /(1-x)
$$

The coefficient of $x^{n}$ in $f^{2}(x)-f\left(x^{2}\right)$ will be twice the number of ways in which $n$ can be represented as the sum of two a's.

* These results were obtained at the Kingston Summer Research Institute of the Canadian Mathematical Congress.

Can. Math. Bull., Vol. 2, No. 2, May 1959 
Thus if the sums of the $a^{\prime} s$ two at a time are to be the same as the sums of the b's two at a time we will have

$$
f^{2}(x)-f\left(x^{2}\right)=g^{2}(x)-g\left(x^{2}\right)
$$

If we let

$$
h(x)=f(x)-g(x)
$$

then (2) can be written in the form

$$
\{f(x)+g(x)\} h(x)=h\left(x^{2}\right)
$$

Now from (4) and (1) we obtain

$$
h(x) / h\left(x^{2}\right)=1-x
$$

Throughout we have $|x|<1$ so that $h\left(x^{2^{k}}\right) \rightarrow h(0)$ as $k \rightarrow \infty$. Further, since $a_{1}=0$ and $h(0)=1$, iteration of (5) yields

$$
h(x)=(1-x)\left(1-x^{2}\right)\left(1-x^{4}\right)\left(1-x^{8}\right) \ldots .
$$

Thus from (1), (3) and (6) we have

$$
f(x)=\frac{1}{2}\left[1 /(1-x)+\prod_{k=0}^{\infty}\left(1-x^{2 k}\right)\right] \text {. }
$$

From (7) it is easy to determine which integers belong to A. Since every integer has a unique representation as a sum of distinct powers of 2 we have

$$
1 /(1-x)=\sum_{n=0}^{\infty} x^{n}=\prod_{k=0}^{\infty}\left(1+x^{2^{k}}\right),
$$

while

$$
\prod_{k=0}^{\infty}\left(1-x^{2^{k}}\right)=\sum_{n=0}^{\infty}(-1)^{\varepsilon(n)} x^{n}
$$

where $\varepsilon(n)$ is the number of summands in the decomposition of $n$ into distinct powers of 2 , i.e. the sum of the digits in the binary representation of $n$.

Combining (7), (8) and (9) we find that A consists precisely of those integers for which $\mathcal{E}(n)$ is even. Thus the sequences $A$ and $B$ are

$$
\begin{aligned}
& A: 0,3,5,6,9,10,12,15, \ldots, \\
& B: 1,2,4,7,8,11,13,14, \ldots .
\end{aligned}
$$


We note that the classes $A$ and $B$ can also be constructed recursively as follows: 0 is in $A$ and 1 in $B$ and if

$$
2^{k} \leqslant x<2^{k+1}
$$

then $x$ and $x-2^{k}$ are in opposite classes.

It is easy to see that our decomposition also leads to a unique decomposition of any arithmetic progression $\{a+n b\}$, $\mathrm{n}=0,1,2, \ldots$ into two classes having the same sums in pairs. Indeed we have only to put $a+n b$ into the same class as was previously assigned to $\mathrm{n}$.

Let us next investigate the possibility of splitting the finite set of integers $0,1,2, \ldots, \mathrm{m}-1$ into two classes in such a way that the sums in pairs in both classes are the same. Using the same definitions for $f(x), g(x)$ and $h(x)$ as before and following a similar procedure we obtain

$$
f(x)+g(x)=1+x+x^{2}+\ldots+x^{m-1}=\left(1-x^{m}\right) /(1-x)
$$

and

$$
h(x)=\frac{1-x}{1-x^{m}} \cdot \frac{1-x^{2}}{1-x^{2}} \cdot \frac{1-x^{4}}{1-x^{4 m}} \cdot \cdots \cdot
$$

Clearly $h(x)$ is a polynomial if and only if $m=2^{k}, k \geqslant 0$. In this case we have

$$
h(x)=(1-x)\left(1-x^{2}\right) \ldots\left(1-x^{2^{k-1}}\right),
$$

with the understanding that this product is 1 when $k=0$, and

$$
f(x)=\frac{1}{2}\left[\left(1-x^{2^{k}}\right) /(1-x)+(1-x)\left(1-x^{2}\right) \ldots\left(1-x^{2 k-1}\right)\right] .
$$

Thus the integers $0,1,2, \ldots, \mathrm{m}-1$ can be split in the required manner if and only if $m=2^{\mathrm{k}}$ and in this case the split must be in accordance with the same rule as was used for the integers $0,1,2, \ldots$.

We next consider a corresponding problem for products instead of sums. We will show that there is a unique way of splitting the positive integers into two classes in such a way that products of pairs of distinct integers from either class occur with the same multiplicities. Suppose then that the positive integers are split into $C$ and $D$ with 


$$
\begin{aligned}
& \mathrm{C}: \mathrm{l}=\mathrm{c}_{1}<\mathrm{c}_{2}<\ldots, \\
& \mathrm{D}: \quad \mathrm{d}_{1}<\mathrm{d}_{2}<\ldots .
\end{aligned}
$$

This time we define the corresponding Dirichlet series

and

$$
C \leftrightarrow F(s)=\sum_{i=1}^{\infty} c_{i}{ }^{-s} \quad(R l(s)>1)
$$

$$
D \leftrightarrow G(s)=\sum_{j=1}^{\infty} d_{j}^{-s} \quad(R l(s)>1) .
$$

Here we have

$$
F(s)+G(s)=\sum_{n=1}^{\infty} n^{-s}=\zeta(s) .
$$

The condition that the products of pairs of distinct c's are the same as products of pairs of distinct d's becomes

$$
F^{2}(s)-F(2 s)=G^{2}(s)-G(2 s) .
$$

Hence if we let

$$
H(s)=F(s)-G(s)
$$

then

$$
H(s) / H(2 s)=(\zeta(s))^{-1} .
$$

Iterating (17) and using $H(\infty)=0$ we obtain

$$
H(s)=[\zeta(s) \zeta(2 s) \zeta(4 s) \zeta(8 s) \ldots]^{-1} .
$$

If we now use the well known Euler identity

$$
\zeta(s)=\prod_{p}\left(1-p^{-s}\right)^{-1} \quad(R 1 s>1),
$$

where the product runs over all primes, we obtain

$$
\begin{aligned}
\text { (20) } \mathrm{H}(\mathrm{s})=\prod_{\mathrm{p}}\left(1-\mathrm{p}^{-\mathrm{s}}\right)\left(1-\mathrm{p}^{-2 \mathrm{~s}}\right)\left(1-\mathrm{p}^{-4 \mathrm{~s}}\right)\left(1-\mathrm{p}^{-8 \mathrm{~s}}\right) \ldots \\
=\prod_{\mathrm{p}}\left(1+\alpha(\mathrm{p}) / \mathrm{p}^{\mathrm{s}}+\alpha\left(\mathrm{p}^{2}\right) / \mathrm{p}^{2 \mathrm{~s}}+\alpha\left(\mathrm{p}^{3}\right) / \mathrm{p}^{3 \mathrm{~s}}+\ldots\right)=\sum_{\mathrm{n}} \alpha(\mathrm{n}) / \mathrm{n}^{\mathrm{s}},
\end{aligned}
$$

where $\alpha(n)$ is the multiplicative function determined by

$$
\alpha\left(\mathrm{p}^{\mathrm{x}}\right)=(-1)^{\varepsilon(\mathrm{r})}
$$


and

$$
\alpha\left(p_{1}^{r_{1}} p_{2}^{r_{2}} \ldots p_{k}^{r_{k}}\right)=\alpha\left(p_{1}^{r_{1}}\right) \alpha\left(p_{2}^{r_{2}}\right) \ldots \alpha\left(p_{k}^{r_{k}}\right)
$$

We are thus led to the following rule for the required classification:

$$
\mathrm{n}=\mathrm{p}_{1}^{\mathrm{r}_{1}} \mathrm{p}_{2}^{\mathrm{r}_{2}} \ldots \mathrm{p}_{\mathrm{k}}^{\mathrm{r}_{\mathrm{k}}}
$$

lies in the first class if and only if the total number of binary digits required to represent the set of numbers $r_{1}, r_{2}, \ldots, r_{k}$ is even. The classification begins

C : $1,6,8,10,12,14,15,18,20,21,22,26,27,28, \ldots$

$\mathrm{D}: 2,3,4,5,7,9,11,13,16,17,19,23,24,25,29,30, \ldots$

It is easy to see that no finite set $1,2, \ldots, n$ can be split into two classes so that the products two at a time are the same for both classes, if $n \geqslant 3$. Indeed, if $n>3$, a consideration of the position of the largest four numbers $n-3, n-2, n-1$ and $n$ already leads to this conclusion. Thus, if $n$ is in the same class as $n-1$ or $n-2$ then the product. of the largest numbers in this class is larger than any product in the second class. On the other hand, if $n-1$ and $n-2$ are in the same class, then their product is larger than that of the largest numbers which could be in the other class, namely $n$ and $n-3$. The case $n=3$ is easily verified independently.

In conclusion we note that the determination of all sets of numbers which have a given set of numbers as sums of pairs appears to be a difficult problem. In particular, in a paper* of J.L. Selfridge and E. Straus which treats a number of related problems, it is conjectured that no three distinct sets of numbers can have the same sum of distinct pairs.

\section{McGill University}

University of Alberta

* On the determination of numbers by their sums of a fixed order, Pacific J. of Math. 8 (1958), pp. 845-856. 